\title{
B33
}

\section{The Robustness and General Applicability of Optimal Resistivity Surveys Designed by Maximising Model Resolution}

\author{
P.B. Wilkinson* (British Geological Survey), J.E. Chambers (British \\ Geological Survey), P.I. Meldrum (British Geological Survey), O. Kuras \\ (British Geological Survey) \& C.J. Munro (British Geological Survey)
}

\section{SUMMARY}

Most optimal survey design algorithms for resistivity imaging have not incorporated prior knowledge of the resistivity of the subsurface. The resulting surveys are optimal for a homogeneous earth, but little investigation has yet been carried out to test whether they are robust, i.e. that they remain optimal when applied to imaging heterogeneous subsurface resistivity distributions. This paper compares a generic survey, which is designed to maximise the estimated model resolution evenly across a homogeneous earth, with specific surveys similarly designed for a number of heterogeneous resistivity distributions. In terms of both the average estimated model resolution and the correlations between the inverted and true resistivity models, the generic and heterogeneous survey designs give near-identical results. This suggests that surveys designed using homogeneous earth approximations are robust in the presence of resistivity heterogeneities and are therefore generally applicable. Traditional dipole-dipole surveys with the same number of measurements do not give such good inverted images, and their degree of optimality (measured either by average resolution or image correlation) is less robust in the presence of heterogeneity. 


\section{Near Surface \\ Geoscience 2012}

\section{Introduction}

Optimal survey design for resistivity imaging seeks to maximise the accuracy, precision and robustness of the inverted subsurface resistivity distribution. Several optimisation algorithms have been proposed (Wilkinson et al., 2012 and references therein) to produce survey designs that outperform traditional measurement arrays (e.g. dipole-dipole). Algorithms that maximise the estimated model resolution can incorporate robustness in the presence of noise (Wilkinson et al., 2012) and produce surveys that are relatively insensitive to the type of inversion constraints used (Loke et al., 2010). The majority of optimisation schemes proposed to date have concentrated on designing surveys for general use and have been based on calculations for a homogeneous earth. But few papers have demonstrated that the optimality of such surveys is maintained when applied to the actual heterogeneous resistivity of the ground. Similarly there are few studies of surveys designed with a degree of prior knowledge of the true resistivity distribution. The exceptions are Stummer et al. (2004), who compared a general optimised survey with an equivalent based on a known resistivity distribution (3 orders-of-magnitude resistivity range, 30 electrodes, 1050 data) and found little difference in the resulting resistivity images. Athanasiou $(2006 ; 2009)$ found some improvement when optimising for a known resistivity distribution but with small numbers of data and electrodes (2 orders-of-magnitude range, 20 electrodes, 150-200 data). Nenna et al (2011) designed a survey with partial prior resistivity knowledge ( $<1$ order-of-magnitude range, 41 electrodes, 200 data), but it was not compared to a general optimised design. In this paper, it is shown that a generic optimised survey, designed by maximising the model resolution of a homogeneous earth using the CR-method (Wilkinson et al., 2012), maintains its optimality when applied to a variety of resistivity distributions.

\section{Method \& Results}

A linearised estimate of the model resolution matrix for the inverse resistivity problem is given by $\mathbf{R} \approx\left(\mathbf{G}^{\mathrm{T}} \mathbf{G}+\mathbf{C}\right)^{-1} \mathbf{G}^{\mathrm{T}} \mathbf{G}$, where $\mathbf{G}$ is the Jacobian matrix comprising the logarithmic sensitivities of the measurements to changes in the model cell resistivities and $\mathbf{C}$ is the constraint matrix. The principal diagonal of $\mathbf{R}$ gives an estimate of $R$, the model resolution of the cells, where $R=0$ is unresolved and $R=1$ is perfectly resolved. All the permitted unique four-electrode measurements form the comprehensive measurement set whose model resolution for a homogeneous earth is shown in Fig. 1(a), calculated for a line of 32 electrodes using the analytic sensitivity function. To investigate arbitrary resistivity models the sensitivity must be calculated numerically, here using a finitedifference method. Its results for a homogeneous half-space are shown in Fig. 1(b) for comparison.

The quality of a survey design is assessed by its relative model resolution $R_{\mathrm{r}}=R_{\text {survey }} / R_{\text {compr. }}$ To generate a survey, the CR-method uses a locally-optimal successive design scheme. This maximises the average relative model resolution across the image of $m$ cells, $S=\Sigma R_{\mathrm{r}} / m$, by direct calculation of the changes in $R_{\mathrm{r}}$ when given measurements are added to the survey. A generic optimised survey was generated using a homogeneous earth model giving $S_{\text {hom }}=0.684$. This was compared to a dipoledipole survey with $a=1-4$ and $n=1-10\left(S_{\text {hom }}=0.597\right)$, and also to surveys optimised for the specific resistivity models being investigated. Each survey had 575 measurements. The forward models used to generate the resistivity data are shown in Fig. 2. They represent (a) resistive inclusions, (b) conductive inclusions, (c) a dipping interface, and (d) a dipping interface with resistive inclusions.

Figure 3 shows the comprehensive and relative model resolution distributions for each of the four models and each of three surveys (dipole-dipole, generic optimised and model optimised). In all four cases the model-optimised surveys were designed using the exact resistivity model. But to investigate a situation with only partial prior information, e.g. knowledge of the dipping interface but not the resistive inclusions, the survey designed for (c) was also applied to (d). In each case, the modeloptimised survey gives the highest average resolution $S_{\max }$. The generic surveys always achieve very similar $S$ values ( $\sim 99 \%$ of $S_{\max }$ ) irrespective of the resistivity distribution and range. In contrast, the dipole-dipole survey yields average resolutions of between $77 \%$ and $81 \%$ of $S_{\max }$ for the heterogeneous models (and 87\% for the homogeneous model). As might be expected, using partial prior knowledge gives an $S$ value between those for the generic and model optimised surveys, but the difference is negligible. The inverted images corresponding to each combination of model and survey 


\section{Near Surface \\ Geoscience 2012}

design are shown in Fig. 4. The quality of the inverted images is measured by $P$, the Pearson correlation coefficient between the image and its corresponding forward model. Note that the model optimised survey does not always give better correlation than the generic optimised survey. This is because the model resolution used in the optimisation process is a linearised estimate. However, in each case the generic and model optimised surveys consistently produce very similar images that are better, both qualitatively and quantitatively, than those produced by the standard dipole-dipole survey.

\section{Conclusions}

The results indicate that surveys designed to maximise average model resolution for a homogeneous earth are robust (very nearly as optimal) when applied to imaging heterogeneous distributions with resistivity ranges of at least 2 orders-of-magnitude. By contrast, standard surveys (e.g. dipole-dipole) do not maintain their degree of optimality with different resistivity models. Since homogeneous and heterogeneous survey designs give similarly optimal results, this also suggests that many such surveys should exist, all providing near-optimal imaging. Although not precluding more optimal or more robust designs that might be found by nonlinear optimisation methods, these results vindicate the use of linear approximations and homogeneous models to enable fast optimal resistivity survey design.

\section{Acknowledgements}

Published with permission of the Executive Director of the British Geological Survey (NERC).

\section{References}

Athanasiou, E.N., Tsourlos, P.I., Papazachos, C.B. and Tsokas, G.N. [2006] Optimizing resistivity array configurations by using a non-homogeneous background model. Proceedings of the 12th EAGE Near Surface Geophysics conference, Helsinki, Finland.

Athanasiou, E.N., Tsourlos, P.I., Papazachos, C.B. and Tsokas, G.N. [2009] Optimizing electrical resistivity array configurations by using a method based on the sensitivity matrix. Proceedings of the 15th EAGE Near Surface Geophysics conference, Dublin, Ireland.

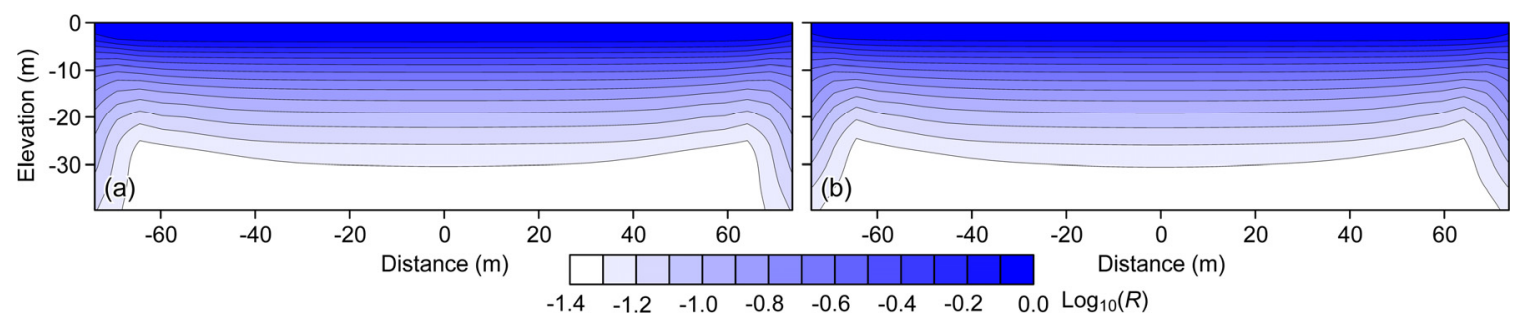

Figure 1 Comprehensive model resolution for a homogeneous earth (a) analytic; (b) finite-difference.

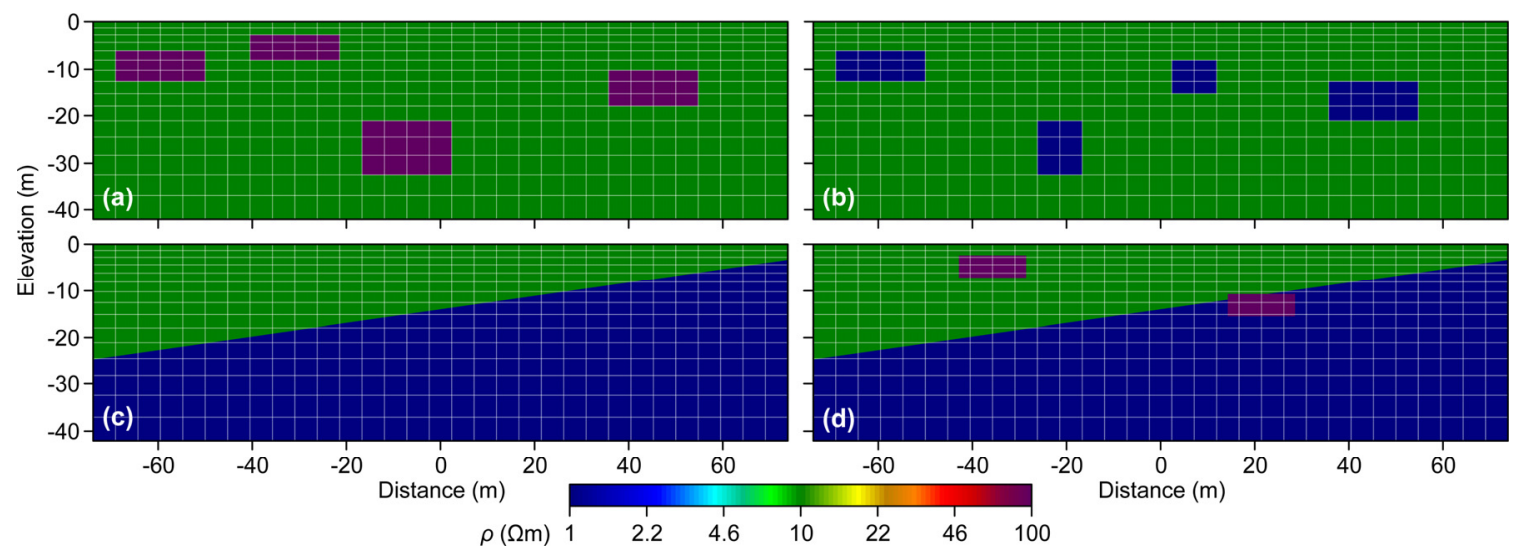

Figure 2 Forward models showing finite-difference model cells. In (c) \& (d) resistivity discontinuities do not align with model cell edges, so each cell resistivity is set to the average value across the cell. 


\section{Near Surface \\ Geoscience 2012}
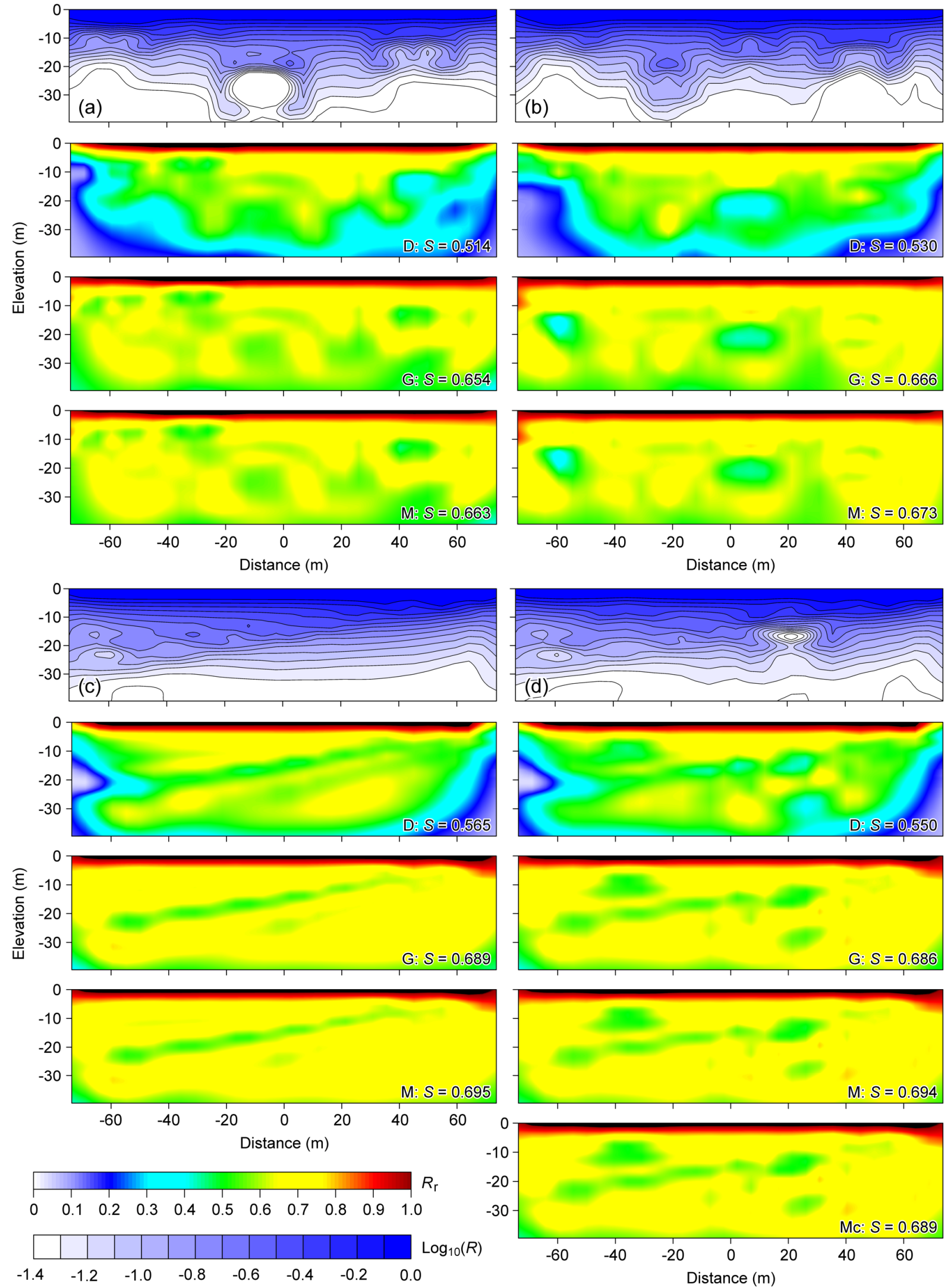

Figure 3(a) Comprehensive model resolution $\mathrm{R}$ for the forward model in Fig. 2(a) and relative model resolution $\mathrm{R}_{r}$ plots for the dipole-dipole $(D)$, generic optimised $(G)$ and resistivity model optimised (M) surveys. (b)-(d) Equivalent resolution plots corresponding to the forward models in Figs. 2(b)(d). Also shown is $\mathrm{R}_{r}$ for model (d) using the resistivity optimised survey for model (c) (denoted Mc). Each $\mathrm{R}_{r}$ plot also displays its average relative resolution $S$. 


\section{Near Surface \\ Geoscience 2012}
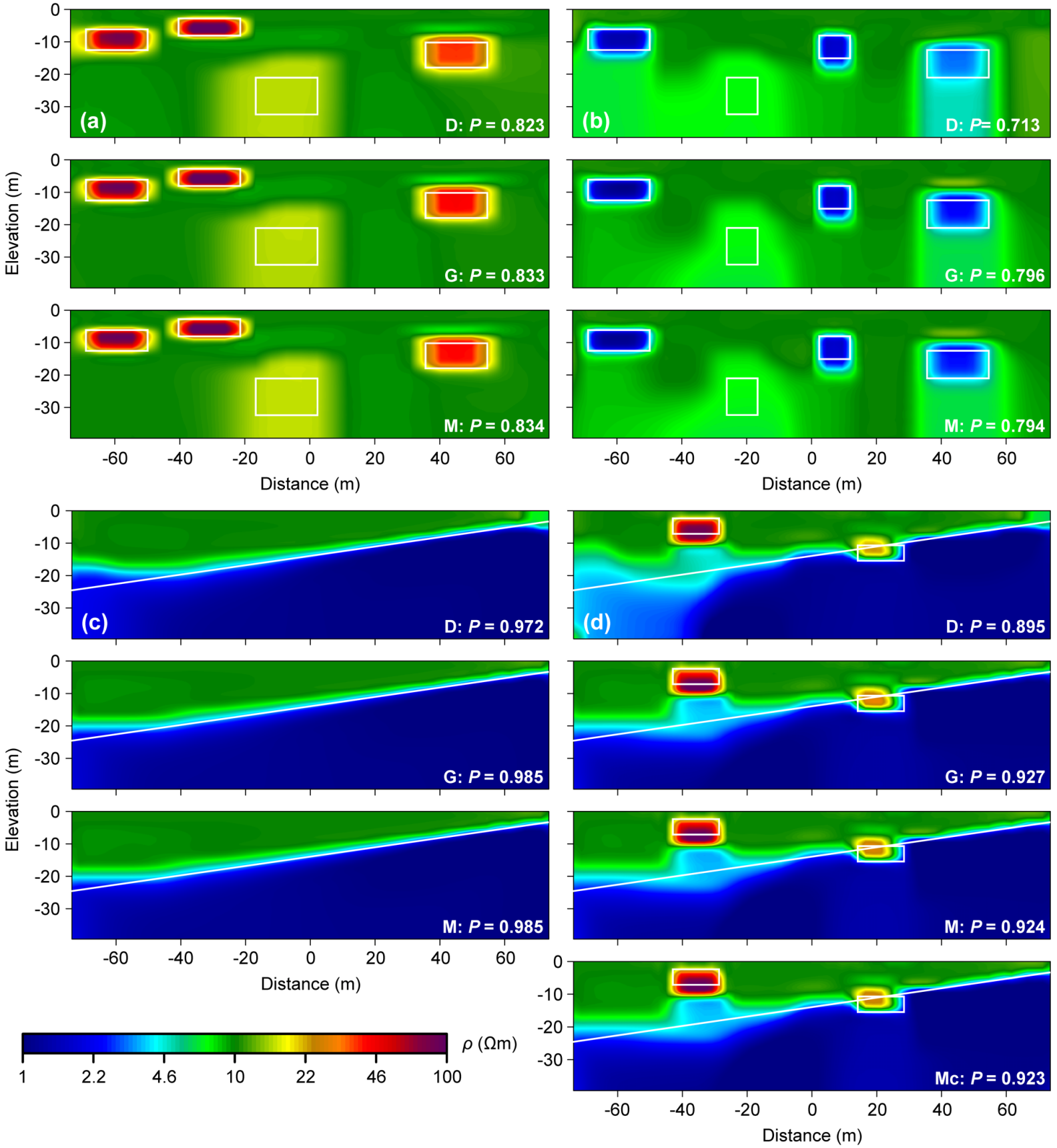

Figure 4(a) Inverted images corresponding to the forward model in Fig. 2(a) for the dipole-dipole $(D)$, generic optimised $(G)$ and resistivity model optimised $(M)$ surveys. (b)-(d) Equivalent inverted images corresponding to the forward models in Figs. 2(b)-(d). Also shown is the inverted image for model (d) using the resistivity optimised survey for model (c) (denoted Mc). Each image also displays the correlation coefficient $P$ between the forward and inverted models.

Loke, M.H., Wilkinson, P.B. and Chambers, J.E. [2010] Parallel computation of optimized arrays for 2-D electrical imaging surveys. Geophysical Journal International, 183(3), 1302-1315.

Nenna, V., Pidlisecky, A. and Knight, R. [2011] Informed experimental design for electrical resistivity imaging. Near Surface Geophysics, 9(5), 469-482.

Stummer, P., Maurer, H. and Green, A.G. [2004] Experimental design: Electrical resistivity data sets that provide optimum subsurface information. Geophysics, 69(1), 120-139.

Wilkinson, P.B., Loke, M.H., Meldrum, P.I., Chambers, J.E., Kuras, O., Gunn, D.A. and Ogilvy, R.D. [2012] Practical aspects of applied optimized survey design for electrical resistivity tomography. Geophysical Journal International, 189(1), 428-440. 